\title{
On the automorphism groups of algebraic bounded domains.
}

\author{
D. Zaitsev
}

February 5, 2008

\section{Contents}

\begin{tabular}{lll}
\hline & Introduction & 1
\end{tabular}

\begin{tabular}{|lll}
2 & Real semi-algebraic sets & 6
\end{tabular}

$\begin{array}{lll}3 \text { A scheme of the proof } & 10\end{array}$

\begin{tabular}{|lll}
\hline 4 & Reflection principle & 10
\end{tabular}

$4.1 \quad$ The case $n \geq 3$. . . . . . . . . . . . . . . . . . . . . . . . 13

4.2 The case $n=2 \ldots \ldots \ldots \ldots \ldots$

4.3 Reflection principle with parameters . . . . . . . . . . . 15

$\begin{array}{lll}5 & \text { The choice of parameters } & 20\end{array}$

6 Defining conditions for $A u t_{a}(D) \quad 21$

6.1 Reduction to a fixed pattern. . . . . . . . . . . . . . 22

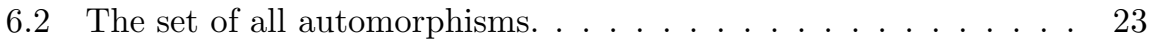

6.3 The image $\imath\left(A u t_{a}(D)\right)$ and the set of associated graphs. . . . . 26

\begin{tabular}{lll}
\hline & Semi-algebraic structures on $A u t_{a}(D)$ & 26
\end{tabular}

\section{Introduction}

Let $D$ be a domain in $\mathbb{C}^{n}$ and $A u t(D)$ be the group of all biholomorphic automorphisms of $D$. Let $v \in D$ be fixed and define the map $C_{v}$ : Aut $(D) \rightarrow D \times G l(n)$ by $f \mapsto\left(f(v), f_{* v}\right)$. The theorem of H. Cartan (see Narasimhan, [7], p. 169) can be stated as follows.

Theorem 1.1 Let $D$ be bounded. Then: 
1. The group Aut $(D)$ possesses a natural Lie group structure compatible with the compact-open topology such that the action $\operatorname{Aut}(D) \times D \rightarrow D$ is real analytic.

2. For all $v \in D$ the map $C_{v}$ is a real-analytic homeomorphism onto its image.

The domains we discuss here are open connected sets defined by finitely many real polynomial inequalities or connected finite unions of such sets. These are the domains in the so-called "semi-algebraic category" defined below (Definition 2.1). For this reason we call them "semi-algebraic domains". In this paper we are interested in the algebraic nature of the image of $A u t(D)$ and its subgroups in $D \times G l(n)$ under the map $C_{v}$.

Example.1. The simplest example of a semi-algebraic domain is the unit disk $D=\{|z|<1\}$. For this domain

$$
\operatorname{Aut}(D)=P G L_{2}(\mathbb{R})_{+}:=\left\{A \in P G L_{2}(\mathbb{R}) \mid \operatorname{det} A>0\right\} .
$$

We see that $A u t(D)$, as a subgroup of $P G L_{2}(\mathbb{R})$, is defined by an inequality and therefore is not an algebraic subgroup. In fact, the group $\operatorname{Aut}(D)$ here does not admit algebraic structure as a Lie group.

To show this, assume that there is a Lie isomorphism $\varphi: P G L_{2}(\mathbb{R})_{+} \rightarrow G$, where $G$ is a real algebraic group. It continues to an isomorphism of complexifications $\varphi^{\mathbb{C}}: P G L_{2}(\mathbb{C}) \rightarrow G^{\mathbb{C}}$. The latter, being a Lie isomorphism between semi-simple complex algebraic groups, is algebraic. Since $G \subset G^{\mathbb{C}}$ is real algebraic, so is its preimage $\left(\varphi^{\mathbb{C}}\right)^{-1}(G)=P G L_{2}(\mathbb{R})_{+}$. On the other hand, $P G L_{2}(\mathbb{R})_{+} \subset P G L_{2}(\mathbb{C})$ is not real Zariski closed. This is a contradiction.

Q. E. D.

Example.2. More generally let $D$ be a bounded homogeneous domain in $\mathbb{C}^{n}$. By the classification theorem of Vinberg, Gindikin and Pyatetskii-Shapiro (see [1], Theorem 6, p. 434), D is biholomorphic to a homogeneous Siegel domain of the 1st or the 2nd kind. Such a domain is defined algebraically in terms of a homogeneous convex cone ([1] ) . Rothaus ([10]) gave a procedure for constructing all homogeneous convex cones. The construction implies that all homogeneous convex cones, and therefore all homogeneous Siegel domains of the 1 st and 2 nd kind, are defined by finitely many polynomial inequalities. The Siegel domains are unbounded but they are birationally equivalent to bounded domains which are also defined by finitely many polynomial inequalities and therefore are semi-algebraic. Thus, $D$ is biholomorphic to a bounded semialgebaic domain.

The automorphism group $A u t(D)$ of a bounded homogeneous domain was discussed by Kaneyuki (see [4]) where he proved in particular that the identity component $\operatorname{Aut}(D)^{0}$ is isomorphic to an identity component of a real algebraic group (价, Theorem.3.2., p.106). Let $x_{0} \in D$ be a fixed point. Since 
$D=\operatorname{Aut}(D) / I \operatorname{so}\left(x_{0}\right)$ and the isotropy group $I s o\left(x_{0}\right)$ is compact, the automorphism group $\operatorname{Aut}(D)$ has finitely many components. Together with the result of Kaneyuki this implies that $A u t(D)$ is isomorphic to an open subgroup of a real algebraic group.

In the above examples the automorphism group $A u t(D)$ is isomorphic to an open subgroup of a real algebraic group. Therefore, it admits a faithful representation. For general domains however the automorphism group does not admit a faithfull representation.

Example.3. Let

$$
D:=\left\{\left.(z, w) \in \mathbb{C}^{2}|| z\right|^{2}+|w|^{2}<1, w \neq 0\right\}
$$

be the unit ball in $\mathbb{C}^{2}$ with a unit disk removed. We consider $D$ as a subset of $\mathbb{P}^{2}$ with homogeneous coordinates $\xi_{0}, \xi_{1}, \xi_{2}, z=\xi_{1} / \xi_{0}, w=\xi_{2} / \xi_{0}$. The automorphism group of $D$ is $A u t(D)=S U(1,1) \times S^{1}$ with the action on $D$ given by

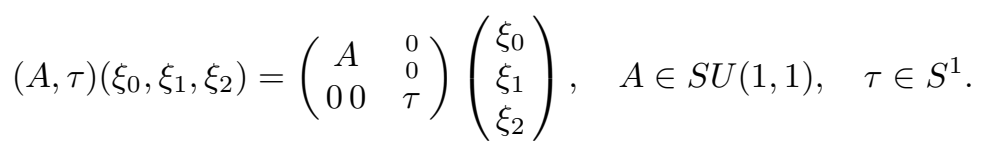

The isomorphism $K: C \mapsto J C J^{-1}$ between $S L_{2}(\mathbb{R})$ and $S U(1,1) \quad(J=$ $\left.\left(\begin{array}{cc}-i & 1 \\ i & 1\end{array}\right)\right)$ yelds an effective action of $S L_{2}(\mathbb{R})$ on $D$. Furthermore, each map $j_{x}: S L_{2}(\mathbb{R}) \rightarrow D, j_{x}(C):=C x$ induces an isomorphism between fundamental groups $\pi_{1}\left(S L_{2}(\mathbb{R})\right)=\pi_{1}(D)=\mathbb{Z}$. This implies that the induced action of a finite covering of $S L_{2}(\mathbb{R})$ on $D$ lifts to an effective action on the finite covering $D^{\prime}$ of $D$ of the same degree. But no covering of $S L_{2}(\mathbb{R})$ admits a faithfull representation (because every such representation factorizes through a representation of $\left.S L_{2}(\mathbb{C})\right)$. On the other hand, the map $(z, w) \mapsto(z, \sqrt[d]{w})$ defines an isomorphism between a finite covering of $D$ of degree $d$ and a bounded semi-algebraic domain $\tilde{D} \subset \mathbb{C}^{2}$.

In this example the group $\operatorname{Aut}(D)$ is not isomorphic to an open subset of an algebraic group. Moreover, even in case it is, the action $\operatorname{Aut}(D) \times D \rightarrow D$ can be "far from algebraic". This phenomena is shown in the following example.

Example.4. Let $F=\mathbb{C} / \Lambda$ be a complex elliptic curve and $\mathcal{P}: F \rightarrow \mathbb{P}^{1}$ the Weierstraß $\mathcal{P}$-function which defines a $2-1$ ramified covering over $P^{1}$. The strip $\{z \in \mathbb{C} \mid c-\epsilon<\operatorname{Im} z<c+\epsilon\}$ covers a "circle strip" $\tilde{D} \subset \mathbb{C} / \Lambda$. Let $D$ be the projection of $\tilde{D}$ on $P^{1}$. If the constant $c$ is generic and $\epsilon$ is small enough, the projection of $\tilde{D}$ is biholomorphic. The real algebraic group $S^{1} \subset \mathbb{C} / \Lambda$ acts by translations on $\tilde{D}$ which yields an effective action on $D$. Since the domain $D$ is bounded by real elliptic curves, it is therefore semi-algebraic. However the action of $S^{1}$ is expressed in terms of the Weierstraß $\mathcal{P}$-function and is not 
algebraic. In fact there is no homomorphism of $S^{1}$ in a real algebraic group $G$ such that the action $S^{1} \times D \rightarrow D$ is given by restrictions of polynomials on $G$. This follows from the classification of 1-dimensional Nash (semi-algebraic) groups given by Madden and Stanton (see [5]).

We see therefore that even in simple cases the class of real algebraic groups and their subgroups is not large enough to describe the group $A u t(D)$ and its action on $D$. Consequently, we consider a larger class of groups where defining inequalities are allowed.

Definition 1.1 1. A Nash function is a real analytic function $f=$ $\left(f_{1}, \ldots, f_{m}\right): U \rightarrow \mathbb{R}^{m}$ (where $U$ is an open semi-algebraic subset of $\mathbb{R}^{n}$ ) such that for each of the components $f_{k}$ there is a nontrivial polynomial $P$ with $P\left(x_{1}, \ldots, x_{n}, f_{k}\left(x_{1}, \ldots, x_{n}\right)\right)=0$ for all $\left(x_{1}, \ldots, x_{n}\right) \in U$.

2. A Nash manifold $M$ is a real analytic manifold with finitely many coordinate charts $\phi_{i}: U_{i} \rightarrow V_{i}$ such that $V_{i} \subset \mathbb{R}^{n}$ is semi-algebraic for all $i$ and the transition functions are Nash (a Nash atlas).

3. A Nash manifold is called affine if it can be Nash (locally closed) imbedded in $\mathbb{R}^{N}$ for some $N$.

4. A Nash group is a Nash manifold with a group operation $(x, y) \rightarrow x y^{-1}$ which is Nash with respect to every Nash coordinate chart.

Remark 1.1 The simplest example of a Nash manifold which is not affine is the quotient $\mathbb{R} / \mathbb{Z}$ with the Nash structure inherited from the standard Nash structure on $\mathbb{R}$. For the classification of such groups in the one-dimensional case see J. J. Madden and C. M. Stanton in [5].

Roughly speaking, the goal of this paper is to prove that the automorphism group $\operatorname{Aut}(D)$ of a semi-algebraic domain $D$ has a natural Nash group structure such that the action $\operatorname{Aut}(D) \times D \rightarrow D$ is also Nash. For this we need a certain non-degeneracy condition on the boundary of $D$. To give the reader a flavour of the main result, we first mention an application for the algebraic domains introduced by Diederich and Fornæss ([2]), for which this condition is automatically satisfied.

Definition 1.2 (see Diederich-Forncess, 国) $A$ domain $D \subset \subset C^{n}$ is called algebraic if there exists a real polynomial $r(z, \bar{z})$ such that $D$ is a connected component of the set

$$
\left\{z \in \mathbb{C}^{n} \mid r(z, \bar{z})<0\right\}
$$

and $d r(z) \neq 0$ for $z \in \partial D$. 
Theorem 1.2 Let $D \subset \subset \mathbb{C}^{n}, n>1$, be an algebraic domain. The group Aut $(D)$ possesses a unique structure of an affine Nash group so that the action Aut $(D) \times D \rightarrow D$ is Nash. For all $v \in D, C_{v}$ :Aut $(D) \rightarrow D \times G l(n)$ is a Nash isomorphism onto its image.

Corollary 1.1 Let $D$ be as in Theorem 1.9. Then the group Aut $(D)$ has finitely many connected components.

Remark 1.2 In general the number of components of Aut $(D)$ can be infinite. For example, let $H:=\{z \in \mathbb{C} \mid \operatorname{Im} z=0\}$ be the upper half-plane and

$$
\hat{H}:=H \cup \bigcup_{n \in \mathbb{Z}} B_{\epsilon}(n),
$$

where $B_{\epsilon}(n)$ is the ball with centre $n$ and radius $\epsilon<1 / 2$. Let $D \subset \mathbb{C}^{2}$ be the union of $H \times H$ and $(H+i) \times \hat{H}$. Then $D$ is biholomorphic to a simply connected bounded domain. The flat pieces of the boundary of $D$ admit canonical foliations $z=$ const and $w=$ const. The latters induce foliations of $D$ of the same form which are preserved by the automorphisms (see Remmert and Stein [9]). By this argument one shows that $\operatorname{Aut}(D)=\mathbb{R} \oplus \mathbb{Z}$.

For the formulation of our main result we need the following condition on $D$, which is automatically satisfied for all bounded domains with smooth boundary, in particular, for all algebraic domains.

Definition 1.3 1. A domain $D \subset \mathbb{C}^{n}$ or its boundary is called Levi-nondegenerate, if there exists a point $x_{0} \in \partial D$ and a neighborhood $U \subset \mathbb{C}^{n}$ of $x_{0}$ such that

$$
D \cap U=\{z \in U \mid \varphi(z)<0\}
$$

for a $C^{2}$-function $\varphi$ with $d \varphi \neq 0$ and such that the Levi form in $x_{0}$

$$
L_{r}\left(x_{0}\right):=\sum_{k, l=1}^{n} \frac{\partial^{2} r}{\partial z_{i} \partial \bar{z}_{j}} d z_{k} \otimes d \bar{z}_{l}
$$

restricted to the holomorphic tangent space of $\partial D$ in $x_{0}$ is non-degenerate;

2. A domain $D \subset \mathbb{C}^{n}$ or its boundary is called completely Levi-nondegenerate, if every boundary point outside a real analytic subset of dimension $2 n-2$ is non-degenerate in the above sense.

Now let $D$ be semi-algebraic and consider the subset $A u t_{a}(D) \subset \operatorname{Aut}(D)$ of all (biholomorphic) automorphisms which are Nash. In the following sense Nash automorphisms are branches of algebraic maps (see Proposition 2.5). 
Definition 1.4 Let $D$ be a domain in $\mathbb{C}^{n}$. A holomorphic map $f \in \operatorname{Aut}(D)$ is a branch of an algebraic map if there exists a complex $n$-dimensional algebraic subvariety $G \subset \mathbb{C}^{n} \times \mathbb{C}^{n}$ which contains the graph of $f$.

The following is the main result of this paper.

Theorem 1.3 Let $D \subset \subset \mathbb{C}^{n}, n>1$, be a semi-algebraic Levi-non-degenerate domain. Then

1. $\operatorname{Aut}_{a}(D)$ is a (closed) Lie subgroup of Aut $(D)$,

2. Aut $t_{a}(D)$ possesses a unique structure of an affine Nash group so that the action Aut $_{a}(D) \times D \rightarrow D$ is Nash.

3. For all $v \in D, C_{v}: \operatorname{Aut}_{a}(D) \rightarrow D \times G l(n)$ is a Nash isomorphism onto its image.

Theorem 1.2 is now a corollary of Theorem 1.3. This is a consequence of the following result of K. Diederich and J. E. Fornæss ([2]).

Theorem 1.4 Let $D \subset \subset \mathbb{C}^{n}$ be an algebraic domain. Then $A_{u} t_{a}(D)=$ Aut $(D)$.

Remark. The proof of Theorem 1.4 makes use of the reflection principle (see S. Pinčuk, [8]) and basic methods of S. Webster ([12]) which are also fundamental in the present paper. Due to the results of K. Diederich and S. Webster ( $\mid 3 \|)$ and of S. Webster ([12]) on the continuation of automorphisms, Theorem 11.3 can be applied to every situation where the automorphisms can be $C^{\infty}$ extended to the boundary.

K. Diederich has informed the author that he and S. Pinčuk recently proved that, under natural non-degeneracy conditions on the boundary, automorphisms of domains are always almost everywhere continuously extendable. Applying this along with the reflection method, one would expect $A u t(D)=\operatorname{Aut}_{a}(D)$ for $D$ a semi-algebraic domain with $\partial D$ completely Levi-non-degenerate. Thus Theorem 1.3 applied to this situation would show that $A u t(D)$ is an affine Nash group acting semi-algebraically on $D$.

Acknowledgement. On this occasion I would like to thank my teacher A. T. Huckleberry for formulating the problem, for calling my attention to the relevant literature and for numerous very useful discussions.

\section{Real semi-algebraic sets}

Here we present some basic properties of semi-algebraic sets which will be used in the proof of Theorem 1.3. For the proofs we refer to Benedetti-Risler [1]. 
Definition 2.1 A subset $V$ of $\mathbb{R}^{n}$ is called semi-algebraic if it admits some representation of the form

$$
V=\bigcup_{i=1}^{s} \bigcap_{j=1}^{r_{i}} V_{i j}
$$

where, for each $i=1, \ldots, s$ and $j=1, \ldots, r_{i}, V_{i j}$ is either $\left\{x \in \mathbb{R}^{n} \mid P_{i j}(x)<0\right\}$ or $\left\{x \in \mathbb{R}^{n} \mid P_{i j}(x)=0\right\}$ for a real polynomial $P_{i j}$.

As a consequence of the definition it follows that finite unions and intersections of semi-algebraic sets are always semi-algebraic. Moreover, closures, boundaries, interiors (see Proposition 2.2) and connected components of semialgebraic sets are semi-algebraic. Further, the number of connected components is finite (see Corollary 2.1). Finally, any semi-algebraic set admits a finite semialgebraic stratification (see Definition 2.3 and Proposition 2.1).

The natural morphisms in the category of semi-algebraic set are semialgebraic maps:

Definition 2.2 Let $X \subset \mathbb{R}^{n}$ and $Y \subset \mathbb{R}^{n}$ be semi-algebraic sets. A map $f: X \rightarrow$ $Y$ is called semi-algebraic if the graph of $f$ is a semi-algebraic set in $\mathbb{R}^{m+n}$.

Definition 2.3 A stratification of a subset $E$ of $\mathbb{R}^{n}$ is a partition $\left\{A_{i}\right\}_{i \in I}$ of E such that

1. each $A_{i}$ (called a stratum) is a real analytic locally closed submanifold of $\mathbb{R}^{n}$;

2. if $\overline{A_{i}} \cap A_{j} \neq \emptyset$, then $\overline{A_{i}} \supset A_{j}$ and $\operatorname{dim} A_{j}<\operatorname{dim} A_{i}$ (frontier condition).

A stratification is said to be finite if there is a finite number of strata and to be semi-algebraic if furthermore each stratum is also a semi-algebraic set.

Proposition 2.1 Every semi-algebraic set $E \subset \mathbb{R}^{n}$ admits a semi-algebraic stratification.

Corollary 2.1 Every semi-algebraic set has a finite number of connected components and each such component is semi-algebraic.

Proposition 2.2 Let $X$ be a semi-algebraic set in $\mathbb{R}^{m}$. Then the closure $\bar{X}$, its interior $\stackrel{0}{X}$, and its boundary $\partial X$ are semi-algebraic sets. 
Using Proposition 2.1, dimension of a semi-algebraic set is defined to be the maximal dimension of its stratum. This is independent of the choice of a finite stratification.

Proposition 2.3 Let $Y \subset \mathbb{R}^{m}$ be a semi-algebraic set of $\operatorname{dim} Y \leq k$. Then it is contained in some real algebraic set $Z$ with $\operatorname{dim} Z \leq k$.

The following results on images and local triviality of semi-algebraic maps will play an important role in the present paper.

Theorem 2.1 (Tarski-Seidenberg) Let $f: X \rightarrow Y$ be a semi-algebraic map. Then the image $f(X) \subset Y$ is semi-algebraic set.

Further we need the Theorem on local triviality (see Benedetti-Risler [1], Theorem 2.7.1, p. 98).

Theorem 2.2 Let $X$ and $Y$ be semi-algebraic sets and let $f: X \rightarrow Y$ be a continuous semi-algebraic map. Fix a finite semi-algebraic partition of $X$, $\left\{X_{1}, \ldots, X_{h}\right\}$. Then there exists

1. a finite semi-algebraic stratification $\left\{Y_{1}, \ldots, Y_{k}\right\}$ of $Y$;

2. a collection of semi-algebraic sets $\left\{F_{1}, \ldots, F_{k}\right\}$ and, for every $i=1, \ldots, k$, a finite semi-algebraic partition $\left\{F_{i 1}, \ldots, F_{i r}\right\}$ of $F_{i}$ (typical fibres);

3. a collection of semi-algebraic homeomorphisms

$$
g_{i}: f^{-1}\left(Y_{i}\right) \rightarrow Y_{i} \times F_{i}, \quad i=1, \ldots, k
$$

such that

(a) the diagram

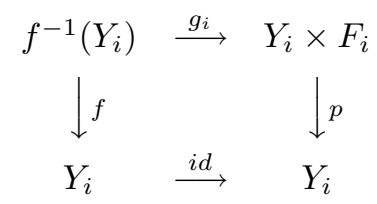

is commutative ( $p: Y_{i} \times F_{i} \rightarrow Y_{i}$ is the natural projection);

(b) for every $i=1, \ldots, k$ and every $h=1, \ldots, r$,

$$
g_{i}\left(f^{-1}\left(Y_{i}\right) \cap X_{h}\right)=Y_{i} \times F_{i h} .
$$

Remark 2.1 In the above setting one says that $f$ is a trivial semi-algebraic map over $Y_{i}$ with typical fibre $F_{i}$ and structure homeomorphisms $g_{i}$. 
Further, we shall use the following proposition from real algebraic geometry (see [1], Proposition 3.2.4).

Proposition 2.4 Let $V \subset \mathbb{R}^{n}$ be a real algebraic variety. The set of singular points is an algebraic set properly contained in $V$.

The following Proposition provides a motivation for Definition 1.4.

Proposition 2.5 The Nash automorphisms $f \in \operatorname{Aut}_{a}(D)$ are branches of algebraic maps.

Proof. Let $f \in \operatorname{Aut}_{a}(D)$ be a Nash automorphism. Then $f$ is holomorphic and semi-algebraic. Every coordinate $f_{j}: D \rightarrow \mathbb{C}$ is also holomorphic and, by Theorem 2.1, semi-algebraic. By Proposition 2.3, there exist real algebraic sets $Z_{j}$ of (real) codimension 2 with $\Gamma_{f_{j}} \subset Z_{j}$. By Proposition 2.4, there exists a regular point $\left(w_{0}, f\left(w_{0}\right)\right) \in \Gamma_{f_{j}}$ where $\Gamma_{f_{j}}$ is locally given by two real polynomials $P_{1}(z, \bar{z}), P_{2}(z, \bar{z})$ or by a complex one $P(z, \bar{z}):=P_{1}(z, \bar{z})+i P_{2}(z, \bar{z})$, such that $d P \neq 0$. The latter property implies that either $\partial P \neq 0$ or $\bar{\partial} P \neq 0$. We can assume that $\partial P \neq 0$, otherwise $P$ can be replaced with $\bar{P}$.

Let $P(z, \bar{z})=P^{\prime}(z)+P^{\prime \prime}(z, \bar{z})$ be a decomposition of $P$ in the holomorphic part $P^{\prime}$ and the remainder $P^{\prime \prime}$ which consists only of terms with non-trivial powers of $\bar{z}$. The part $P^{\prime}$ is not zero because $d P^{\prime}=\partial P \neq 0$. We wish to prove that $\Gamma_{f_{j}}$ is locally defined by the holomorphic polynomial $P^{\prime}(z)$. The identity

$$
P\left(w, f_{j}(w), \bar{w}, \overline{f_{j}(w)}\right) \equiv 0
$$

for all $w$ near $w_{0}$ implies in particular the vanishing of the Taylor coefficients of $w^{k}$ for all multi-indices $k$. But these coefficients are just

$$
\frac{1}{|k| !} \frac{\partial^{|k|} P^{\prime}\left(w, f_{j}(w)\right)}{\partial w^{k}}
$$

and their vanishing means the vanishing of $P^{\prime}\left(z, f_{j}(z)\right)$.

Thus the graph $\Gamma_{f_{j}}$ is locally defined by a holomorphic polynomial $P_{j}$ and the polynomials $P_{1}, \ldots, P_{n}$ define the $n$-dimensional algebraic variety required in the definition of a branch of an algebraic map.

Q. E. D.

We shall also make frequent use of Chevalley's theorem on constructible sets (see Mumford [6], p. 72).

Definition 2.4 $A$ subset $A \in \mathbb{C}^{n}$ is called constructible if it is a finite union of locally closed complex algebraic subvarieties. 
Remark 2.2 Constructible sets are semi-algebraic.

Theorem 2.3 (Chevalley) Let $X$ and $Y$ be affine varieties and $f: X \rightarrow Y$ any morphism. Then $f$ maps constructible sets in $X$ to constructible sets in $Y$.

\section{A scheme of the proof}

The proof of Theorem 1.3 can be divided in two steps. The essential ingredient for the first step is the method of S. Webster (see 112]) based on the reflection principle (see S. Pinčuk, 81). We use it to construct an appropriate family of graphs of automorphisms from $A_{u t}(D)$. In fact we construct a constructible family $F$ which fibres contain automorphisms from $A u t_{a}(D)$. This is carried out in sections 1 and 4.3 .

In the second step we show using the family constructed in the first step that the set $C_{v}\left(\operatorname{Aut}_{a}(D)\right)$ is Nash. Taking a neighborhood where $C_{v}\left(A u t_{a}(D)\right)$ is not empty and closed and taking its pullback in $\operatorname{Aut}(D)$ we obtain a neighborhood where $A u t_{a}(D)$ is closed, which implies statement 1. in Theorem 1.3. In fact we prove that the exact family of graphs

$$
\Gamma:=\left\{\left(C_{v}(f), w, f(w) \mid f \in \operatorname{Aut}_{a}(D), w \in \mathbb{C}^{n}\right\}\right.
$$

is Nash. If we identify $A u t_{a}(D)$ with $C_{v}\left(A u t_{a}(D)\right), \Gamma$ is the graph of the action $A_{u t}(D) \times D \rightarrow D$. This proves statement 3 . To obtain statement 2., we observe that group operation can be defined semi-algebraically in terms of $\Gamma$. Here we use the theory of semi-algebraic sets and their morphisms.

\section{Reflection principle}

Let $D \subset \subset \mathbb{C}^{n}$ be a semi-algebraic Levi-non-degenerate domain. By Proposition 2.2, the boundary $\partial D$ is semi-algebraic. By Proposition 2.3, there exists a real algebraic set $H$ of dimension $2 n-1$ which contains $\partial D$. Let $H_{i}$ be irreducible components of $H$ of dimension $2 n-1$. By Definition 1.3, the Levi form of some component of $H$, let say of $H_{1}$, is not everywhere degenerate.

To every irreducible hypersurface $H_{i}$ we associate a real Zariski open set $U_{i} \subset \mathbb{C}^{n}$ and a real polynomial $r_{i}(z, \bar{z})$ with $H_{i} \cap U_{i}=\left\{r_{i}=0\right\} \cap U_{i}$ and $d r \neq 0$ on $U_{i}$. By Proposition 2.4, such $U_{i}$ 's and $r_{i}$ 's exist.

Let $f \in \operatorname{Aut}_{a}(D)$ be any fixed map which is, by Proposition 2.5 a branch of an algebraic map and $V \subset \mathbb{C}^{n} \times \mathbb{C}^{n}$ be the corresponding $n$-dimensional algebraic subvariety which contains the graph of $f$. We wish to extend $f$ in a neighborhood of a boundary point $x \in H_{1}$. Outside a proper complex algebraic 
subvariety $V^{\prime} \subset V$ the variety $V$ defines $f$ and $f^{-1}$ as possibly multiple-valued algebraic maps. Since $\operatorname{dim}_{\mathbb{C}} V=n, \operatorname{dim}_{\mathbb{C}} V^{\prime} \leq n-1$. Since $\operatorname{dim}_{\mathbb{R}} H_{1}=2 n-1$, there exists a point $x \in H_{1} \cap U_{1}$ and a neighborhood $U \subset U_{1}$ of $x$ such that $V$ is a trivial covering over $U$ and all sections of this covering define biholomorphic maps onto their images. One of these maps coincides with $f$ over $D \cap U$. This map yields the desired extension of $f$. Since $f$ is an automorphism of $D$, it maps $H_{1} \cap U$ into $\partial D$.

Notation. Let $i=i(f)$ be such that $f\left(H_{1} \cap U\right) \subset H_{i}$. Let $w_{0} \in H_{1} \cap U$ be an arbitrary point such that for $w_{0}^{\prime}=f\left(w_{0}\right)$ one has $d r_{i}\left(w_{0}^{\prime}\right) \neq 0$.

We use the notation

$$
r:=r_{1}, r^{\prime}:=r_{i}, H:=H_{1} \text {, and } H^{\prime}:=H_{i} .
$$

Then $f(H \cap U) \subset H^{\prime}$ and we have a relation

$$
r^{\prime}(f(z), \bar{f}(\bar{z}))=g(z, \bar{z}) r(z, \bar{z}),
$$

where $g(z, \bar{z})$ is real analytic.

Let $z=x+i y$, where $x$ and $y$ are real coordinate vectors. Since the functions in (i1) are given by power series in $(x, y)$, they are still defined for complex vectors $x$ and $y$ near $w_{0}$. This is equivalent to varying $z$ and $\bar{z}$ independently. The relation (i1) persists:

$$
r^{\prime}(f(z), \bar{f}(\bar{w}))=g(z, \bar{w}) r(z, \bar{w}) .
$$

Now we consider the spaces $Z:=\mathbb{C}^{n}, Z^{\prime}:=\mathbb{C}^{n}, W:=\mathbb{C}^{n}$ and $W^{\prime}:=\mathbb{C}^{n}$ and define the complexifications $\mathcal{H} \subset Z \times \bar{W}$ and $\mathcal{H}^{\prime} \subset Z^{\prime} \times \bar{W}^{\prime}$ by

$$
r(z, \bar{w})=0, \quad r^{\prime}\left(z^{\prime}, \bar{w}^{\prime}\right)=0 .
$$

The so-called Segre complex varieties associated to the points $w \in W$ are defined by

$$
Q_{w}=\{z \in Z \mid r(z, \bar{w})=0\} .
$$

Since $g(z, \bar{w})$ is holomorphic for $z$ and $w$ near $w_{0}$, we see from (2) that the map $f^{\mathbb{C}}:=f \times \bar{f}$ takes $\{z\} \times Q_{z}$ into $\left\{z^{\prime}\right\} \times Q_{z^{\prime}}^{\prime}$, where $z^{\prime}=f(z)$. Hence, the family of complex hypersurfaces $\{z\} \times Q_{z}$ is invariantly related to $H$.

Since $r(z, \bar{z})$ is real, we have

$$
r(z, \bar{w})=\bar{r}(\bar{w}, z)=\overline{r(w, \bar{z})},
$$

so that $z \in Q_{w} \Longleftrightarrow w \in Q_{z}$. Also $z \in Q_{z} \Longleftrightarrow z \in H$. Since $r$ is real and 
$d r=\partial r+\bar{\partial} r$ does not vanish at $w_{0}$ (resp. $r^{\prime}$ is real and $d r^{\prime}=\partial r^{\prime}+\bar{\partial} r^{\prime}$ does not vanish at $w_{0}^{\prime}$, we have

$$
\partial r(z, \bar{w}) \neq 0, \quad \partial r^{\prime}\left(z^{\prime}, \bar{w}^{\prime}\right) \neq 0
$$

for $z$ and $w$ near $w_{0}$ and for $z^{\prime}$ and $w^{\prime}$ near $w_{0}^{\prime}$.

The relation (4) implies that $Q_{w}$ is non-singular in $z$ if $(z, \bar{w})$ is near $\left(w_{0}, \bar{w}_{0}\right)$. Let $\pi_{z}(\bar{w})$ denote the complex tangent space $T_{z} Q_{w}$ as an element in the grassmanian $G_{n, n-1}$. It follows that $\pi_{z}$ is an antiholomorphic map from $\{z\} \times Q_{z}$ to $G_{n, n-1}$. S. Webster proves the following fact (12, p. 55, Lemma 1.1):

Lemma 4.1 The antiholomorphic map $\pi_{z}(\bar{w})$ is locally invertible near the points of $H$ where the Levi form is non-degenerate.

Since the Levi form of $U \cap H$ is non-degenerate, and a biholomorphic map preserves this property, the Levi form of $H^{\prime}$ is non-degenerate around $w_{0}^{\prime}=$ $f\left(w_{0}\right) \in H^{\prime}$. By Lemma 4.1, $\pi_{z}$ and $\pi_{z^{\prime}}^{\prime}$ are locally invertible for $(z, \bar{w})$ near $\left(w_{0}, \bar{w}_{0}\right)$ and $\left(z^{\prime}, \bar{w}^{\prime}\right)$ near $\left(w_{0}^{\prime}, \bar{w}_{0}^{\prime}\right)$.

The first step of Webster's method is to describe the map $f$ between $Q_{z}$ and $Q_{z^{\prime}}^{\prime}$. We have seen that $f$ takes $Q_{w}$ into $Q_{w^{\prime}}^{\prime}, w^{\prime}=f(w)$. We take $w \in Q_{z}$. Then all $Q_{w}$ 's pass through the point $z$ and all $Q_{w^{\prime}}^{\prime}$ 's through $z^{\prime}$. Therefore the differential $f_{* z} \in G l(n)$ takes $T_{z} Q_{w}$ into $T_{z^{\prime}} Q_{w^{\prime}}^{\prime}$, i.e. $\pi_{z}(\bar{w})$ into $\pi_{z^{\prime}}\left(\bar{w}^{\prime}\right)$. These considerations mean that the restriction $\left.f\right|_{Q_{z}}$ can be decomposed as follows $([12]$, p. 56$)$ :

$$
w \stackrel{\pi_{z}}{\longmapsto} T_{z} Q_{w} \stackrel{J_{n-1}\left(f_{* z}\right)}{\longmapsto} T_{z^{\prime}} Q_{w^{\prime}}^{\prime} \stackrel{\pi_{z^{\prime}}^{-1}}{\longmapsto} w^{\prime},
$$

where the second map $J_{n-1}\left(f_{* z}\right): G_{n, n-1} \rightarrow G_{n, n-1}^{\prime}$ is the natural map between grassmanians which is induced by the differential $f_{* z}: T_{z} Z \rightarrow T_{z^{\prime}} Z^{\prime}$.

The decomposition (5) implies that for $w \in Q_{z}$ and $w^{\prime}=f(w), z^{\prime}=f(z)$ and $q=f_{* z} \in G l(n)$ the following relation is satisfied:

$$
\pi_{z^{\prime}}^{\prime}\left(\bar{w}^{\prime}\right)=J_{n-1}(q)\left(\pi_{z}(\bar{w})\right) .
$$

This relation expresses the restriction $\phi:=\left.f\right|_{Q_{z}}$ in terms of parameters $\left(z, z^{\prime}, q\right) \in Z \times Z^{\prime} \times G l(n)$. This is of great importance for our parametrization of the graphs of elements of $A u t_{a}(D)$. Hence we underline this fact by introducing the notation

$$
\phi\left(\bar{z}, \bar{z}^{\prime}, \bar{q}\right): Q_{z} \rightarrow Q_{z^{\prime}}^{\prime}
$$

for $\phi:=\left.f\right|_{Q_{z}}$. We write the conjugate variables for arguments of $\phi$ in order to emphasize that $\phi$ depends holomorphically on them.

The idea of the second step is to express the map $f$ in terms of restrictions $\left.f\right|_{Q_{z}}$. This is done separately for $n=2$ and $n \geq 3$. 


\subsection{The case $n \geq 3$}

Let $z_{0} \in U \cap Q_{w_{0}}$ be any point. Lemma 4.1 gives points $v_{1}, \ldots, v_{n} \in Q_{z_{0}}$ near $w_{0}$ such that all $Q_{v_{j}}$ are non-singular and transverse in $z_{0}$ and the algebraic curve $\gamma$, defined by

$$
r\left(z, \bar{v}_{1}\right)=\cdots=r\left(z, \bar{v}_{n-1}\right)=0,
$$

is transverse to $Q_{w_{0}}$. Every $w$ near $w_{0}$ lies in some $Q_{z}, z \in \gamma$, namely for $z \in \gamma \cap Q_{w}$. Therefore, to describe $f(w)$ we need only to consider restrictions $\left.f\right|_{Q_{z}}, z \in \gamma$.

Given the values $z^{\prime}=f(z)$ and differentials $q=f_{* z} \in G l(n)$, the map $\left.f\right|_{Q_{z}}$ is determined by (6). Since $\gamma \subset Q_{v_{1}}$, the values $z^{\prime}=f(z)$ along $\gamma$ are determined, in turn, due to (6) by parameters $\left(v_{1}, v_{1}^{\prime}, l_{1}\right)=\left(v_{1}, f\left(v_{1}\right), f_{* v_{1}}\right)$. Namely, we use the map $\phi$ in (6) and set

$$
z^{\prime}=\phi\left(\bar{v}_{1}, \bar{v}_{1}^{\prime}, \bar{l}_{1}\right)(z) .
$$

Further, the differentials $f_{* z}$ along $\gamma$ can be expressed in terms of parameters $\left(v_{1}, v_{1}^{\prime}, l_{1}\right)=\left(v_{1}, f\left(v_{1}\right), f_{* v_{1}}\right)$. Consider the differential 1-forms

$$
\theta_{\alpha}=\partial r\left(z, \bar{v}_{\alpha}\right) .
$$

They define a frame in the cotangent spaces. Let

$$
\left\{Y_{j}=Y_{j}\left(z, \bar{v}_{1}, \ldots, \bar{v}_{n-1}, \bar{w}\right), j=1, \ldots, n\right\}
$$

be the dual vector field frame. This frame has rational coefficients in the variables $\left(z, \bar{v}_{1}, \ldots, \bar{v}_{n-1}, \bar{w}\right)$ and satisfies the conditions

$\left.\begin{array}{c}Y_{1} \text { is transverse to } Q_{v_{1}} \text { and tangent to } Q_{v_{2}}, \\ Y_{2} \text { is transverse to } Q_{v_{2}} \text { and tangent to } Q_{v_{1}}, \\ Y_{3}, \ldots, Y_{n} \text { are tangent to } Q_{v_{1}} \cap Q_{v_{2}}\end{array}\right\}$

Similar differential 1-forms $\theta_{\alpha}^{\prime}$ and frame vector fields $Y_{j}^{\prime}$ are constructed for $H^{\prime}$. Relative to these two frame fields

$$
f_{* z} Y_{l}=\sum q_{l j} Y_{j}^{\prime},
$$

where

$$
\left[q_{l j}\right]=\left[\begin{array}{ccc}
q_{11} & 0 & 0 \\
0 & q_{22} & 0 \\
q_{1 \beta} & q_{2 \beta} & q_{\alpha \beta}
\end{array}\right]
$$

$\alpha, \beta=3, \ldots, n$.

The functions $q_{11}, q_{1 \beta}, q_{\alpha \beta}$ are determined by values of $f$ along $Q_{v_{1}}$, i.e. by $\phi\left(v_{1}, v_{1}^{\prime}, l_{1}\right)$ (where $v_{1}^{\prime}=f\left(v_{1}\right)$ and $l_{1}=f_{* v_{1}}$ ). Similarly, $q_{22}$ and $q_{2 \beta}$ are 
determined by $\phi\left(v_{2}, v_{2}^{\prime}, l_{2}\right)$. These dependencies can be expressed by relations

$$
\left.\begin{array}{rl}
q_{11} & =\theta_{1}^{\prime}\left(\phi_{* z}\left(v_{1}, v_{1}^{\prime}, l_{1}\right) Y_{1}\right), \\
q_{1 \beta} & =\theta_{1}^{\prime}\left(\phi_{* z}\left(v_{1}, v_{1}^{\prime}, l_{1}\right) Y_{\beta}\right), \\
q_{\alpha \beta} & =\theta_{\alpha}^{\prime}\left(\phi_{* z}\left(v_{1}, v_{1}^{\prime}, l_{1}\right) Y_{\beta}\right), \\
q_{22} & =\theta_{2}^{\prime}\left(\phi_{* z}\left(v_{2}, v_{2}^{\prime}, l_{2}\right) Y_{2}\right), \\
q_{2 \beta} & =\theta_{2}^{\prime}\left(\phi_{* z}\left(v_{2}, v_{2}^{\prime}, l_{2}\right) Y_{\beta}\right), \\
q_{12} & =q_{21}=q_{\alpha 1}=q_{\alpha 2}=0 .
\end{array}\right\}
$$

Thus, the map $f$ is completely determined by parameters $v_{j} \in V_{j}:=\mathbb{C}^{n}, v_{j}^{\prime} \in$ $V_{j}^{\prime}:=\mathbb{C}^{n}$ and $l_{i} \in L_{j}:=G l(n), j=1, \ldots, n$.

\subsection{The case $n=2$}

In case $n=2$ there are no frames with properties (10) and another construction (12], p. 58) is needed. By Lemma 4.1, two points $\zeta_{1}, \zeta_{2} \in Q_{w_{0}}$ can be chosen such that $Q_{\zeta_{1}}$ and $Q_{\zeta_{2}}$ are non-singular and transverse in $w_{0}$. Then choose $v_{1} \in Q_{\zeta_{1}}$ and $v_{2} \in Q_{\zeta_{2}}$ such that each $Q_{v_{j}}$ is non-singular in $\zeta_{j}$ and transverse to $Q_{w_{0}}$ there. Now fix $v_{1}$ and $v_{2}$ and let $z_{1}$ and $z_{2}$ move along $Q_{v_{1}}$ and $Q_{v_{2}}$ respectively. For $z_{1}$ and $z_{2}$ near $\zeta_{1}$ and $\zeta_{2}$, it follows that $Q_{z_{1}}$ and $Q_{z_{2}}$ are still transverse near $w_{0}$ and intersect each other in a single point $w$ there. Conversely, for given $w$ near $w_{0}, Q_{w}$ intersects each $Q_{v_{j}}$ transversely in a point $z_{j}$ near $\zeta_{j}$. In this way a local biholomorphic correspondence between $w \in W\left(:=\mathbb{C}^{n}\right)$ and $\left(z_{1}, z_{2}\right) \in Q_{v_{1}} \times Q_{v_{2}}$ is obtained. It is defined by relations

$$
\begin{gathered}
r\left(w, \bar{z}_{j}\right)=0 \quad\left(\Longleftrightarrow z_{j} \in Q_{w}\right), \quad j=1,2 ; \\
r\left(v_{j}, \bar{z}_{j}\right)=0 \quad\left(\Longleftrightarrow z_{j} \in Q_{v_{j}}\right) .
\end{gathered}
$$

Further, set $w_{0}^{\prime}:=f\left(w_{0}\right), \zeta_{j}^{\prime}:=f\left(\zeta_{j}\right), v_{j}^{\prime}:=f\left(v_{j}\right)$. All transverse properties are preserved by the biholomorphic map $f$. Again, one obtains a local biholomorphic correspondence between $w^{\prime} \in W^{\prime}$ and $\left(z_{1}^{\prime}, z_{2}^{\prime}\right) \in Q_{v_{1}^{\prime}} \times Q_{v_{2}^{\prime}}$, which is defined by

$$
\begin{array}{ll}
r^{\prime}\left(w^{\prime}, \bar{z}_{j}^{\prime}\right)=0 \quad\left(\Longleftrightarrow w^{\prime} \in Q_{z_{j}^{\prime}}^{\prime}\right) ; \\
r^{\prime}\left(v_{j}^{\prime}, \bar{z}_{j}^{\prime}\right)=0 \quad\left(\Longleftrightarrow z_{j}^{\prime} \in Q_{v_{j}^{\prime}}^{\prime}\right) .
\end{array}
$$

Since the Segre varieties $Q_{z}$ are invariant with respect to $f$, if $z_{j}^{\prime}:=f\left(z_{j}\right)$, one obtains the corresponding point $w^{\prime}=f(w)$. Thus, $f$ can be decomposed in the following way:

$$
W \longrightarrow Q_{v_{1}} \times Q_{v_{2}} \stackrel{f \times f}{\longrightarrow} Q_{v_{1}^{\prime}}^{\prime} \times Q_{v_{2}^{\prime}}^{\prime} \longrightarrow W^{\prime}
$$


The middle map here is in fact $\left.f\right|_{Q_{v_{1}}} \times\left. f\right|_{Q_{v_{2}}}$ which is equal to $\phi\left(\bar{v}_{1}, \bar{v}_{1}^{\prime}, \bar{l}_{1}\right) \times$ $\phi\left(\bar{v}_{2}, \bar{v}_{2}^{\prime}, \bar{l}_{2}\right)$, where $\phi$ is the map (7) and $l_{j}:=f_{* v_{j}}$. In other words we have a relation between $z_{j}$ and $z_{j}^{\prime}$ :

$$
z_{j}^{\prime}=\phi\left(\bar{v}_{j}, \bar{v}_{j}^{\prime}, \bar{l}_{j}\right)\left(z_{j}\right)
$$

Thus, $f$ is completely determined by parameters $v_{j} \in V_{j}:=\mathbb{C}^{n}, v_{j}^{\prime} \in V_{j}^{\prime}:=$ $\mathbb{C}^{n}$ and $l_{i} \in L_{j}:=G l(n), j=1,2$.

\subsection{Reflection principle with parameters}

The local construction recalled in previous paragraph is in fact global because of its algebraic nature. The map $f$ was locally expressed in terms of parameters $\left(v, v^{\prime}, l\right)=\left(v, f(v), f_{* v}\right) \in P$, where $v:=\left(v_{1}, \ldots, v_{n}\right)$ and

$$
P:=\prod_{j=1}^{n}\left(V_{j} \times V_{j}^{\prime} \times L_{j}\right) .
$$

Using the same algebraic relations globally, we shall obtain a constructible family $F \subset P \times W \times W^{\prime}$ such that the graph $\Gamma_{f}$ is an open subset of the closure of the fibre $\overline{F_{p}}$ for generic $v$ and $p=\left(v, f(v), f_{* v}\right)$ (this will be made precise below).

We start with construction of a constructible family for the map

$$
\phi\left(z, z^{\prime}, q\right): Q_{z} \rightarrow Q_{z^{\prime}}^{\prime}
$$

in (7). For this we consider the constructible subset

$$
\Phi \subset \overline{G l(n)} \times \bar{Z} \times W \times \bar{Z}^{\prime} \times W^{\prime}
$$

defined by relations (3), (4), and (6). The relations (4) provide the existence of $\pi_{z}(\bar{w})$ and $\pi_{z^{\prime}}^{\prime}\left(\bar{w}^{\prime}\right)$ respectively. Furthermore, we have seen that

$$
\left(\overline{f_{* z}}, \bar{z}, w, \overline{f(z)}, f(w)\right) \in \Phi
$$

for $(z, \bar{w}) \in \mathcal{H}$ near $\left(w_{0}, \bar{w}_{0}\right)$, and $\pi_{z}$ and $\pi_{z^{\prime}}$ are local invertible there (Lemma 4.1). To provide this local invertibility "globally", we assume, changing if necessary to a smaller constructible subset of $\Phi$, that

$$
\operatorname{det} \frac{\partial \pi_{z}(\bar{w})}{\partial \bar{w}} \neq 0, \quad \operatorname{det} \frac{\partial \pi_{z^{\prime}}^{\prime}\left(\bar{w}^{\prime}\right)}{\partial \bar{w}^{\prime}} \neq 0 .
$$

The set $\Phi$ defines now a family of possibly multiple-valued maps

$$
\phi\left(\bar{z}, \bar{z}^{\prime}, \bar{q}\right): Q_{z} \rightarrow Q_{z^{\prime}}^{\prime} .
$$

Consider the complexifications $\mathcal{H} \subset Z \times \bar{W}$ and $\mathcal{H}^{\prime} \subset Z^{\prime} \times \bar{W}^{\prime}$. Then $\Phi$ is a subset in $\overline{G l(n)} \times \overline{\mathcal{H}} \times \overline{\mathcal{H}}^{\prime}$. 
Lemma 4.2 The projection $\delta: \Phi \rightarrow \overline{G l(n)} \times \bar{Z}^{\prime} \times \overline{\mathcal{H}}$ has finite fibres and is locally biholomorphic.

Proof. We need to prove that $w^{\prime} \in \delta^{-1}\left(\bar{q}, \bar{z}^{\prime}, \bar{z}, w\right)$ depends locally holomorphically on $\left(\bar{q}, \bar{z}^{\prime}, \bar{z}, w\right)$. For this it is enough to observe, that, by $(19), \pi_{z^{\prime}}(\bar{w})$ is locally invertible and, by (6) $\bar{w}^{\prime}=\left(\pi_{z^{\prime}}^{\prime}\right)^{-1}\left(J_{n-1}(q)\left(\pi_{z}(\bar{w})\right)\right)$. Since the above fibres are constructible, they are finite.

Q. E. D.

In the following let $\phi$ denote the multiple-valued map defined by $\Phi$. Since every value of $\phi$ is, by Lemma 4.2, locally holomorphic in $w$, we can discuss its differential $\phi_{*}=\phi\left(\bar{q}, \bar{z}^{\prime}, \bar{z}, w\right)_{*}$ which is also possibly multiple-valued.

For the construction of the required family we need to consider auxiliary parameter spaces $A:=Z \times Z^{\prime} \times G l(n)$ for $n>2$ and $A:=\left(Z \times Z^{\prime}\right)^{2}$ for $n=2$. Let $F \subset \bar{A} \times P \times W \times W^{\prime}$ be the constructible subset defined by relations (3), (6), (8), (9) and (12) in case $n>2$ and by (13), (14), (15), (16) and (18) in case $n=2$.

Passing if necessary to a constructible subset, we can require that in case $n>3$ all $Q_{v_{j}}$ 's and $Q_{w}$ are transverse in $z$ and all $Q_{v_{j}^{\prime}}^{\prime}$ 's and $Q_{w^{\prime}}^{\prime}$ are transverse in $z^{\prime}$. In case $n=2$ we require that each $Q_{v_{j}}$ is transverse to $Q_{w}$ in $z_{j}$ and $Q_{z_{j}}$ 's are transverse in $w$ and, similarly, each $Q_{v_{j}^{\prime}}^{\prime}$ is transverse to $Q_{w^{\prime}}^{\prime}$ in $z_{j}^{\prime}$ and $Q_{z_{j}^{\prime}}^{\prime}$ 's are transverse in $w^{\prime}$.

Further, by Theorem 2.3 of Chevalley, the projection $\pi(F)$ of $F$ on $P \times$ $W \times W^{\prime}$ is also constructible. We don't have in general a local biholomorphic property as in Lemma 4.2 for $\pi(F)$, but we still can prove the finiteness:

Lemma 4.3 The projection $\sigma: \pi(F) \rightarrow P \times W$ has finite fibres.

Proof. Let fix $(p, w) \in P \times W$. Let $\left(p, w, w^{\prime}\right) \in F$ be any point. By the construction of $\pi(F)$, there exist points $a \in A$ such that $\left(\bar{a}, p, w, w^{\prime}\right) \in F$.

Case $n>2$ Let $a=\left(z, z^{\prime}, q\right)$. We constructed $F$ such that $Q_{v_{1}}, \ldots, Q_{v_{n-1}}$ and $Q_{w}$ are transversal in $z$. Then, by (3) and (8), the set of possible $z \in Z$ is discrete and therefore finite. Further, by (9) and (12), only finitely many $z^{\prime \prime}$ s and $q$ 's are possible. Here we use Lemma 4.2. Now $w^{\prime} \in W^{\prime}$ is determined by (6), which implies finiteness of the set of $w^{\prime \prime} \mathrm{s}$.

Case $n=2$ Let $a=\left(z_{1}, z_{1}^{\prime}, z_{2}, z_{2}^{\prime}\right)$. By definition of $F_{2}, Q_{w}$ and $Q_{v_{j}}$ are transverse in $z_{j}$. Then there are only finitely many possible intersections $z_{j}$. By (18), the number of possible $z_{j}^{\prime}$ 's is also finite. Finally, since $Q_{z_{j}^{\prime}}^{\prime}$ are transverse in $w^{\prime}$, the number of possible $w^{\prime \prime}$ s is also finite.

Q. E. D. 
Now let $f \in \operatorname{Aut}_{a}(D)$ be fixed and $H=H_{1}, H^{\prime}=H_{i}$ for $i=i(f)$ (we defined $i(f)$ by the condition $f\left(H_{1} \cap U\right) \subset H_{i}$ for some open $U \subset \mathbb{C}^{n}$ with $\left.H_{1} \cap U \neq \emptyset\right)$. By our construction, $\left(v, f(v), f_{* v}, w, f(w)\right) \in \pi(F)$ for all $(v, w)$ in some open subset $U_{1} \subset V \times W$. Here we wish to point out that the family $\pi(F)$ depends on the index $i=i(f)$. To include all automorphisms $f \in A u t_{a}(D)$, we just consider the finite union of $\pi(F)_{i} \subset P \times W \times W^{\prime}$ for all possible $i=i(f), f \in A_{u t}(D)$ and denote it again by $F$.

The set $F$ is constructible, i.e. a finite union of locally closed algebraic subvarieties. It follows that the set

$$
E(f):=\left\{(v, w) \in D^{n+1} \mid\left(v, f(v), f_{* v}, w, f(w)\right) \notin F\right\}
$$

is analytically constructible, i.e. a finite union of locally closed analytic subvarieties. If $(v, w)$ is outside the closure of $E(f)$, we have $(p, w, f(w)) \in F$ for $p=\left(v, f(v), f_{* v}\right) \in P$. This means that the graph $\Gamma_{f}:=\{(w, f(w)) \mid w \in D\}$ lies in the closure of the fibre $F_{p}$.

Now we wish to prove main result of this section.

Proposition 4.1 Let $P, W$ and $W^{\prime}$ be as above. There exist constructible subsets $F \subset P \times W \times W^{\prime}$ and $E \subset P \times V \times W$ such that

1. The projection $\sigma: F \rightarrow P \times W$ has finite fibres;

2. For every fixed $f \in \operatorname{Aut}_{a}(D)$ there exists a proper subset $E(f) \subset D^{n+1}$, such that for all $(v, w) \in\left(D^{n+1}\right) \backslash E(f)$ and $p=\left(v, f(v), f_{* v}\right) \in P$ one has $(p, w, f(w)) \in F$, the graph $\Gamma_{f}$ is a subset of the closure of the fibre $F_{p}$ and $E(f) \subset E_{p}$;

3. $E_{p}$ is of complex codimension at least 1 in $V \times W$.

We need the following lemma.

Lemma 4.4 Let $A, B$ and $C \subset A \times B$ be constructible subsets of arbitrary algebraic varieties. Then the fibrewise closure of $C$ in $A \times B$, i.e. the union of closures of the fibres $C_{a}:=(\{a\} \times B) \cap C, a \in A$ is constructible.

The proof is based on the following fact (see Mumford, [6], Corollary 1, p.71). Recall that a morphism is dominating if its image is dense.

Proposition 4.2 Let $X$ and $Y$ be two complex algebraic varieties, $f: X \rightarrow Y$ be a dominating morphism and $r=\operatorname{dim} X-\operatorname{dim} Y$. Then there is a nonempty open set $U \subset Y$ such that, for all $y \in U, f^{-1}(y)$ is a nonempty "pure" $r$-dimensional set, i.e. all its components have dimension $r$. 
Proof of Lemma 4.4. We first observe that given two constructible subsets $C_{1}, C_{2} \subset A \times B$ which have constructible fibrewise closures, the union $C=C_{1} \cup$ $C_{2}$ has also this property. Changing to locally closed irreducible components, we can assume that $A, B$ and $C$ are irreducible algebraic varieties.

Now we prove the statement by induction on dimension of $A$. In case $\operatorname{dim} A=0$ the fibrewise closure of $C$ is just the closure of $C$ which is constructible.

Let $\pi: \bar{C} \rightarrow A$ denote the projection of the closure $\bar{C}$ on $A$. We can assume $\pi$ to be dominant, otherwise $A$ is replaced by the closure of $\pi(\bar{C})$ which has a smaller dimension. Now we apply Proposition 4.2 to the projection $\pi$ and obtain an open subset $U \subset A$, such that the fibre's over $U$ have pure $\operatorname{dimension} \operatorname{dim} C-$ $\operatorname{dim} A$. We have a partition $A=U \cup(A \backslash U)$ of $A$ and the corresponding partition $C=\left(C_{1} \cup C_{2}\right)\left(C_{1}:=C \cap(U \times B), C_{2}:=C \cap((A \backslash U) \times B)\right)$. By the above observation, it is enough to prove the statement for $C_{1}$ and $C_{2}$ separately. The statement for $C_{2}$ follows by induction, because $\operatorname{dim}(A \backslash U)<\operatorname{dim} A$. Therefore we can assume $A=U$.

Now we consider the irreducible components $C_{i}$ of $\bar{C} \backslash C, \operatorname{dim} C_{i}<\operatorname{dim} C$. If $S_{i}:=\overline{\pi\left(C_{i}\right)} \neq A$ for some $i$, then we replace $A$ by $A \backslash S_{i}$ and correspondingly $C$ by $C \cap \pi^{-1}\left(A \backslash S_{i}\right)$. Thus we may assume that $\pi: C_{i} \rightarrow A$ is dominaiting for all $i$. Then we can apply Proposition 4.2 to every $C_{i}$ and obtain a number of open sets $U_{i} \subset A$. Let $U$ be the intersection of all $U_{i}$ 's. Since $A$ is irreducible, $U$ is not empty. Again, proceeding by induction, we can reduce the statement to the case $A=U$. But in this case the fibres of $\bar{C}$ are of pure dimension $\operatorname{dim} C-\operatorname{dim} A$ and the fibres of $\bar{C} \backslash C$ have smaller dimension. This implies that the fibrewise closure of $C$ coincides with the usual closure $\bar{C}$ which is constructible.

Q. E. D.

Proof of Proposition 4.1. Statement 1 follows from Lemma 4.3. It follows from the local Webster's construction (section 4$)$ that $(p, w, f(w)) \in F(p=$ $\left.\left(v, f(v), f_{* v}\right)\right)$ for all $(v, w)$ in an open subset $U \subset D^{n+1}$. This means that $U$ lies in the complement of the "exceptional set" $E(f)$. Let $\Omega(f):=D^{n+1} \backslash E(f)$, i.e.

$$
\Omega(f)=\left\{(v, w) \in D^{n+1} \mid\left(v, f(v), f_{* v}, w, f(w)\right) \in F\right\} .
$$

The set $E$ must be globally defined independently of any automorphism $f \in \operatorname{Aut}_{a}(D)$. For this it is necessary to define $\Omega(f)$ in another way. Changing if necessary to a constructible subset of $F$, we may assume that the projection $\sigma_{p}: F_{p} \rightarrow W$ is locally biholomorphic and $\Omega(f)$ still contains an open subset $U \subset D^{n+1}$. Then the differentials $\frac{\partial w^{\prime}}{\partial w}$ are certainly defined. We now define the family $F^{\prime} \subset F \times G l(n)$ of differentials by adding values of $\frac{\partial w^{\prime}}{\partial w}$ :

$$
F^{\prime}:=\left\{\left(p, w, w^{\prime}, q\right) \in F \times G l(n) \mid q=\frac{\partial w^{\prime}}{\partial w}\right\}
$$


This is a constructible set and we have $\left(v, f(v), f_{* v}, w, f(w), f_{* w}\right) \in F^{\prime}$ for $(v, w)$ in some open set $U \subset D^{n+1}$. Now we write the definition of $\Omega(f)$ in the form:

$$
\begin{gathered}
\Omega(f):=\left\{(v, w) \in V \times W \mid \text { for } v^{\prime}=f(v), l=f_{* v}, w^{\prime}=f(w):\right. \\
\left.\left(v, v^{\prime}, l, w, w^{\prime}\right) \in F\right\} .
\end{gathered}
$$

Now we define a set $\Omega$ which contains $\Omega(f)$ for all $f \in A u t_{a}(D)$ :

$$
\begin{array}{r}
\Omega:=\left\{\left(p, v, w, v^{\prime}, w^{\prime}, l\right) \in P \times V \times W \times V^{\prime} \times W^{\prime} \times G l(n) \mid\right. \\
\left.\forall j:\left(p, v_{j}, v_{j}^{\prime}, l_{j}\right) \in F^{\prime} \wedge\left(p, w, w^{\prime}\right) \in F \wedge\left(v, v^{\prime}, l, w, w^{\prime}\right) \in F\right\} .
\end{array}
$$

For $f \in \operatorname{Aut}_{a}(D),(v, w) \in U$, and $p=\left(v, f(v), f_{*} v\right)$, we have

$$
\left(v, w, f(v), f(w), f_{* v}\right) \in \Omega_{p}
$$

Let $\Omega^{\prime}$ be the fibrewise closure of $\Omega$, i.e. the union of all closures of $\Omega_{p}, p \in P$. By Lemma 4.4, $\Omega^{\prime}$ is constructible. Finally, we define $E \subset P \times V \times W$ to be the projection of $\Omega^{\prime} \backslash \Omega$ on $P \times V \times W$. By Theorem 2.3 of Chevalley, $E$ is constructible.

We now wish to prove that every fibre $E_{p}$ is of codimension at least 1 . For this we note that for $p \in P$ fixed the projection of $\Omega_{p}$ on $V \times W$ has finite fibres, i.e. $\operatorname{dim} \Omega_{p} \leq \operatorname{dim}(V \times W)$. This implies $\operatorname{dim}\left(\Omega^{\prime} \backslash \Omega\right)_{p}<\operatorname{dim}(V \times W)$ and $E_{p}$ is of codimension at least 1 as required in statement 3 .

We take now any $(v, w) \in D^{n+1}$ outside $E(f)$ and set $p=\left(v, f(v), f_{* v}\right)$. For the proof of statement 2, consider $f \in \operatorname{Aut}_{a}(D)$, take $(v, w) \in D^{n+1} \backslash E(f)$, and set $p=\left(v, f(v), f_{*} v\right)$. We shall prove that $E(f) \subset E_{p}$. Let $\left(v_{0}, w_{0}\right) \in E(f)$ be any point. If $(v, w) \in U$, we have

$$
\left(v, f(v), f_{* v}, w, f(w), f_{* w}\right) \in F^{\prime},
$$

which implies

$$
\left(p, v, w, f(v), f(w), f_{* v}\right) \in \Omega .
$$

Here $(v, w) \in U$ is arbitrary. Since $f$ is holomorphic, we have this property globally for all $(v, w) \in D^{n+1}$ if we replace $\Omega$ with its fibrewise closure $\Omega^{\prime}$. In particular, we have

$$
\left(v_{0}, w_{0}, f\left(v_{0}\right), f\left(w_{0}\right), f_{* v_{0}}\right) \in \Omega_{p}^{\prime} .
$$

Since $\left(v_{0}, w_{0}\right) \in E(f)$, the point

$$
\left(v_{0}, f\left(v_{0}\right), f_{* v_{0}}, w_{0}, f\left(w_{0}\right)\right)
$$

does not lie in $F$. This implies that

$$
\left(v_{0}, w_{0}, f\left(v_{0}\right), f\left(w_{0}\right), f_{* v_{0}}\right)
$$

does not lie in $\Omega_{p}$ and then it is in $\overline{\Omega_{p}} \backslash \Omega_{p}$. This means $\left(v_{0}, w_{0}\right) \in E_{p}$, which is required. The proof of Proposition 4.1 is finished.

Q. E. D. 


\section{The choice of parameters}

In the previous section we proved the existence of a constructible algebraic family $F \subset P \times W \times W^{\prime}$ with the property that for all $f \in A u t_{a}(D)$ there exists a point $p \in P$ such that

$$
\Gamma_{f} \subset \overline{F_{p}} .
$$

The goal of this section is to choose for every $f$ appropriate parameter $p$ with this property and obtain a map $\imath$ from $A u t_{a}(D)$ in the corresponding parameter space $P$. The first idea is to take some generic $v \in V\left(:=V_{1} \times \cdots \times V_{n}=\mathbb{C}^{n^{2}}\right)$ and to define $p=\left(v, f(v), f_{* v}\right)$. If $(v, w) \notin E(f)$, Proposition 4.1 yields the required property (20). However, if we wish to define a global map $A u t_{a}(D) \rightarrow P$, the condition $(v, w) \notin E(f)$ must be satisfied for all $f \in \operatorname{Aut}_{a}(D)$. Unfortunately, this is not true in general. It is therefore necessary to take sufficiently many points $\left(v_{\mu}, w_{\mu}\right) \in V \times W$ instead of one $(v, w)$, such that $\left(v_{\mu}, w_{\mu}\right) \notin E(f)$ is always true at least for one $\mu$. In fact, we prove the following Proposition.

Proposition 5.1 There exists a natural number $N$, a constructible subset $F \subset$ $P^{N} \times W \times W^{\prime}$ and a collection of points $v_{1}, \ldots, v_{m} \in D, m=n N$, such that

1. the projection $\sigma: F \rightarrow P^{N} \times W$ has finite fibres,

2. for all $f \in$ Aut $_{a}(D)$ the graph $\Gamma_{f}$ is a subset of the closure $\overline{F_{\imath(f)}}$, where the map $\imath: \operatorname{Aut}(D) \rightarrow P^{N}$ is given by $\imath(f)=$ $\left(v_{1}, f\left(v_{1}\right), f_{* v_{1}}, \ldots, v_{m}, f\left(v_{m}\right), f_{* v_{m}}\right)$.

Remark 5.1 Once the set $v_{1}, \ldots, v_{m}$ is chosen, we can add to it finitely many other $v$ 's and not change the statement of Proposition 5.1.

Before we start with the proof we need a technical lemma.

Lemma 5.1 Let $A, B, C \subset A \times B$ be constructible sets and every fibre $C_{a}:=$ $\{b \in B \mid(a, b) \in C\}$ be of codimension at least one. Then there exists a finite number of points $b_{\mu} \in B, \mu=1, \ldots, s$ such that for every $a \in A$ there is a point $b_{\mu} \notin C_{a}$.

Proof. We first prove the statement for $A$ a locally closed irreducible subvariety by induction on dimension of $A$. If $\operatorname{dim} A=0$, the statement is obvious. Assume it to be proven for $\operatorname{dim} A<d$. By definition of constructible sets, $C$ is a finite union of locally closed subvarieties $C_{\alpha}=U_{\alpha} \cap F_{\alpha}$ where $U_{\alpha}$ are Zariski open and $F_{\alpha}$ are closed subvarieties. The subvarieties $F_{\alpha}$ are not open, otherwise a fibre $C_{a}$ would contain an open subset. So we can choose a point

$$
\left(a_{0}, b_{0}\right) \in\left(\cap_{\alpha} U_{\alpha}\right) \backslash\left(\cup_{\alpha} F_{\alpha}\right)
$$


The set $A^{\prime}$ of points $a \in A$, such that $b_{0} \in C_{a}$, is the projection on $A$ of the intersection $\left(A \times\left\{b_{0}\right\}\right) \cap C$, which is constructible. There is an entire neighborhood of $a_{0}$ in the complement and, hence, $A^{\prime}$ has lower dimension than $A$. Now we use induction for all irreducible components of the closure $\overline{A^{\prime}}$. This yields a number of points $b_{\mu}$. These points together with $b_{0}$ satisfy the required condition.

To prove the statement in case $A$ is constructible we note, that $A$ is by Definition 2.4 a finite union of locally closed $A_{\alpha}$ 's. For every $A_{\alpha}$ with $C_{\alpha}:=$ $\left(A_{\alpha} \times B\right) \cap C$ the statement of Lemma gives a finite set of points $b_{\mu}$. The union of these finite sets for all $\alpha$ satisfies the required property.

Q. E. D.

Proof of Proposition 5.1. Now we apply Lemma 5.1 to our situation. Let $P^{\prime}$ be the constructible subset of all parameters $p \in P$ such that $E_{p} \subset V \times W$ is of codimension at least 1. Then we set in Lemma 5.1 $A:=P^{\prime}, B:=V \times W$ and $C:=E \cap\left(P^{\prime} \times V \times W\right)$. The statement of Lemma yields a number of points

$$
\left(v^{(\mu)}, w_{\mu}\right) \in D^{n+1}, \quad \mu=1, \ldots, N .
$$

For every $f \in \operatorname{Aut}_{a}(D)$ and $(v, w) \notin E(f)$ we have by condition 3 in Proposition 4.1, $E(f) \subset E_{p}$ for $p=\left(v, f(v), f_{* v}\right)$. Then for some $\mu=1, \ldots, N$ we have $\left(v^{(\mu)}, w_{\mu}\right) \notin E_{p}$, i.e. $\left(v^{(\mu)}, w_{\mu}\right) \notin E(f)$ and, by condition 2 in Proposition 4.1, $(p, w, f(w)) \in F$. We obtain $m=N n$ points $v_{1}, \ldots, v_{m}$.

Now we construct the required family $F$ to be the union of the sets $F_{\mu}$ defined by

$$
F_{\mu}:=\left\{\left(p_{1}, \ldots, p_{N}, w, w^{\prime}\right) \in P^{N} \times W \times W^{\prime} \mid\left(p_{\mu}, w, w^{\prime}\right) \in F\right\},
$$

Statement 1 in Proposition 5.1 follows from condition 1 in Proposition 4.1 . Let $\imath: \operatorname{Aut}(D) \rightarrow P^{N}$ be the map defined by

$$
\imath(f):=\left(v_{1}, f\left(v_{1}\right), f_{* v_{1}}, \ldots, v_{m}, f\left(v_{m}\right), f_{* v_{m}}\right) .
$$

It is in fact a product of Cartan maps $C_{v}: f \mapsto\left(f(v), f_{* v}\right)$ and is therefore a homeomorphism onto its image. Statement 2 in Proposition 5.1 follows now from the above choice of $v_{j}$ 's.

Q. E. D.

\section{Defining conditions for $A u t_{a}(D)$}

In Proposition 5.1 we constructed a map $\imath: \operatorname{Aut}(D) \rightarrow P^{N}$. Our goal here is to give semi-algebraic description of the image $\imath\left(A u t_{a}(D)\right)$ and to prove the following Proposition. 
Proposition 6.1 The image $\imath\left(\operatorname{Aut}_{a}(D)\right)$ and the set of all graphs

$$
\Gamma:=\left\{(\imath(f), w, f(w)) \mid f \in \operatorname{Aut}_{a}(D) \wedge w \in D\right\}
$$

are semi-algebraic.

\subsection{Reduction to a fixed pattern.}

In Proposition 5.1 we obtained a constructible family $F \subset P^{N} \times W \times W^{\prime}$. Our goal now is to find a stratification of $P^{N} \times W$ such that $F$ has a simplier form over each stratum. This is done by applying Theorem 2.2 on local triviality of semi-algebraic morphisms.

To simplify the notation we shall write $P$ for $P^{N}$. We first consider the projection $\sigma: F \rightarrow P \times W$. Since we are interested only in points over $P \times D \subset P \times W$, we write $F \subset P \times D \times W^{\prime}$ for the intersection with $P \times D \times W^{\prime}$. Since $D$ is semi-algebraic, $F$ is semi-algebraic. The projection $\sigma: F \rightarrow P \times D$ is a continuous semi-algebraic map (see Definition 2.2) and we can apply Theorem 2.2 on local triviality. Theorem 2.2 yields a finite semi-algebraic stratification $\left\{Y_{1}, \ldots, Y_{h}\right\}$ of $P \times D$ (see Definition 2.3), a collection of semi-algebraic typical fibres $\left\{E_{1}, \ldots, E_{h}\right\}$ and a collection of semi-algebraic structural homeomorphisms

$$
\tilde{e}_{i}: Y_{i} \times E_{i} \rightarrow \sigma^{-1}\left(Y_{i}\right), \quad i=1, \ldots, h
$$

(the $\tilde{e}_{i}$ 's here are the inverses of the $g_{i}$ 's in Theorem 2.2.). By statement 1 in Proposition 5.1, every typical fibre $E_{i}$ is finite.

The semi-algebraic stratification $\left\{Y_{i}\right\}$ of the product $P \times D$ defines a stratification of every fibre $\{p\} \times D$. This stratification depends on $p \in P$ and the qualitative picture (e.g. the number of open strata) can also depend on $p$. How-

ever, by changing to a partition of $P$ we reduce this general case to the case of fixed stratification of $\{p\} \times D$, a fixed pattern.

For this we apply Theorem 2.2 again to the projection $\rho: P \times D \rightarrow P$ and partition $\left\{Y_{1}, \ldots, Y_{h}\right\}$ of $P \times D$. We obtain a finite semi-algebraic stratification $\left\{P_{1}, \ldots, P_{r}\right\}$ of $P$, a collection of semi-algebraic typical fibres $\left\{G_{1}, \ldots, G_{r}\right\}$, for every $l=1, \ldots, r$ a finite semi-algebraic partition $\left\{G_{l 1}, \ldots, G_{l h}\right\}$ of $G_{l}$ and a collection of semi-algebraic structural homeomorphisms

$$
g_{l}: P_{l} \times G_{l} \rightarrow \rho^{-1}\left(P_{l}\right), \quad l=1, \ldots, r,
$$

such that

$$
g_{l}\left(P_{l} \times G_{l i}\right)=\left(\rho^{-1}\left(P_{l}\right)\right) \cap Y_{i}, \quad l=1, \ldots, r, \quad i=1, \ldots, h .
$$




\subsection{The set of all automorphisms.}

Here we discuss the set of all automorphisms of $D$ the graphs of which are contained in the closures of fibres of our family $F$. By condition 1 in Proposition 5.1, only finitely many automorphisms can be contained in the closure of a fixed fibre. On the other hand, a fixed automorphism can be contained in closures of a multitude of fibres.

Without loss of generality we assume, that $\left\{G_{l 1}, \ldots, G_{l h}\right\}$ is a finite semialgebraic stratification of $G_{l}$ and $G_{l i}$ are connected (see Proposition 2.1 and Corollary 2.1).

Now for fixed $p \in P_{l}$ we wish to determine if the fibre $F_{p} \subset D \times W^{\prime}$ is related to some $f \in \operatorname{Aut}(D)$. Our procedure for doing this is semi-algebraic: over the fixed decomposition $D=\sqcup_{i} G_{i l}$ (in fact only over open $G_{i l}$ 's) we consider the pieces of $F_{p}$, determined by the trivialization of it with typical fibres $E_{i}$. The condition that certain of these pieces fit together to form a graph of an automorphism proves to be semi-algebraic.

Among the strata $G_{l i}, i=1, \ldots, h$ we choose the open one's, which are assumed to be $G_{l i}, i=1, \ldots, t, t \leq h$. By Proposition 5.1, the projection $\sigma: F \rightarrow P \times D$ has finite fibres so the typical fibres $E_{i}$ are all finite. Let us fix a $t$-tuple $e=\left(e_{1}, \ldots, e_{t}\right) \in E:=E_{1} \times \cdots \times E_{t}$. The number of possible $t$-tuples is finite. Further, we define the maps $\xi_{e, p}$ over each open $\left(Y_{i}\right)_{p}:=\{w \in D \mid$ $\left.(p, w) \in Y_{i}\right\} \subset D$ by

$$
\left(p, w, \xi_{e, p}(w)\right)=\tilde{e}_{i}\left(p, w, e_{i}\right), \quad i=1, \ldots, t,
$$

where $\tilde{e}_{i}: Y_{i} \times E_{i} \rightarrow \sigma^{-1}\left(Y_{i}\right)$ are the trivialization morphisms in (23).

Proposition 6.2 Let $P_{l}$ and $e \in E$ be fixed. The set $P_{e, l}$ of all parameters $p \in P_{l}$ such that the map $\xi_{e, p}$ extends to a biholomorphic automorphism from Aut $_{a}(D)$ is semi-algebraic.

We begin with three lemmas. The first one is a semi-algebraic version of Lemma 4.4 on constructible sets.

Lemma 6.1 Let $A, B$ and $C \subset A \times B$ be semi-algebraic sets. Then the "fibrewise" closure of $C$, i.e. the union of closures of the fibres $C_{a}:=(\{a\} \times B) \cap C$, $a \in A$ is semi-algebraic.

Proof. Let $X:=A \times B$ and consider the partition $X_{1}:=C, X_{2}:=(A \times$ $B) \backslash C$ of $X$. Apply Theorem 2.2 to the projection of $X$ on $A$. To obtain the closures of fibres we take the closures of typical fibres $F_{i 1}$ in $F_{i}$ and their images in $X$ under the structural trivializing homeomorphisms. The images are semi- 
algebraic by Theorem 2.1. The union of these images for all $i$ is semi-algebraic and is exactly the "fibrewise" closure of $C$.

Q. E. D.

Lemma 6.2 Let $A, B$ and $C, D \subset A \times B$ be semi-algebraic sets. Then the set of $a \in A$ such that $C_{a} \subset D_{a}$ is semi-algebraic.

Proof. The complement of the required set in $A$ coincides with the projection on $A$ of the difference $C \backslash D$. The difference of semi-algebraic set is semi-algebraic, the projection is semi-algebraic by the Tarski-Seidenberg theorem (Theorem 2.1).

Q. E. D.

Lemma 6.3 Let $A, B, C, E \subset A \times B$ and $G \subset E \times C$ be semi-algebraic sets. Then the set of all $a \in A$, such that for all $b \in E_{a}$ the fibre $G_{(a, b)}$ consists of exactly one point, is also semi-algebraic.

Proof. We apply the Theorem 2.2 on local trivialization to the projection of $G$ on $E$. This yields a partition $\left\{Y_{i}\right\}$ of $E$. The set $E^{\prime} \subset E$ of one-point fibres $G_{(a, b)}$ is then the finite union of $Y_{i}$ 's such that the corresponding typical fibres $F_{i}$ consist of one point. It follows that $E^{\prime}$ is semi-algebraic. The required set in $A$ coincides with the set of $a \in A$ such that $C_{a} \subset E_{a}^{\prime}$. The latter set is semi-algebraic by Lemma 6.2.

Q. E. D.

Proof of Proposition 6.2. We first consider the condition that $\xi_{e, p}$ extends to a well-defined continuous map on $D$. This means that for any point $w \in$ $\overline{\left(Y_{i}\right)_{p}} \cap \overline{\left(Y_{j}\right)_{p}}, i, j=1, \ldots, t$, the limits of graphs of $\xi_{e, p}$ over $\left(Y_{i}\right)_{p}$ and $\left(Y_{j}\right)_{p}$ coincide over $w$ and consist of one point.

For every stratum $G_{l s}, G_{l i}, G_{l j}, s=1, \ldots, h, i, j=1, \ldots, t$, with

$$
G_{l s} \subset \overline{\left(Y_{i}\right)_{p}} \cap \overline{\left(Y_{j}\right)_{p}}
$$

we write these conditions in a form

$$
\left.\begin{array}{l}
\text { i) } \overline{\overline{\Gamma_{i}(p)}} \cap\left(B(p) \times W^{\prime}\right)=\overline{\Gamma_{j}(p)} \cap\left(B(p) \times W^{\prime}\right), \\
\text { ii) } \quad \forall w \in B(p): \#\left(\overline{\Gamma_{i}(p)} \cap\left(\{w\} \times W^{\prime}\right)\right)=1
\end{array}\right\} \text {, }
$$

where

$$
B(p):=g_{l}\left(\{p\} \times G_{l s}\right) \subset\{p\} \times D
$$

and $\Gamma_{i}(p):=\tilde{e}_{i}\left(\left(Y_{i}\right)_{p} \times\left\{e_{i}\right\}\right)$ is the graph of $\xi_{e, p}$ over $\left(Y_{i}\right)_{p}$. 
Now, by Lemmas 6.1 and 6.2, the set $\left\{p \in P_{l} \mid i\right)$ in (27) is satisfied $\}$ is a semi-algebraic subset of $P_{l}$. For the condition ii) in (27) we set in Lemma 6.3 $A:=P^{\prime}, B:=D, C:=W^{\prime}$,

$$
E:=\left\{(p, w) \in P^{\prime} \times D \mid w \in B(p)\right\}
$$

and

$$
G:=\left\{\left(p, w, w^{\prime}\right) \in E \times W^{\prime} \mid w \in \overline{\Gamma_{i}(p)} \cap\left(B(p) \times W^{\prime}\right)\right\} .
$$

Then, by Lemma 6.3, the set $\left\{p \in P_{l} \mid i i\right)$ in (27) is satisfied $\}$ is a semi-algebraic subset of $P_{l}$.

Without loss of generality, $i$ ) and $i i)$ are satisfied for $p \in P_{l}$. Thus, the closures of graphs of $\xi_{e, p}$ over $\{p\} \times D$ yield well-defined maps $\xi_{p}: D \rightarrow W^{\prime}$ (We do not know yet, whether or not these maps are continuous).

The next condition on $\xi_{e, p}$ is

$$
\xi_{e, p}(D)=D
$$

which is, by Lemma 6.2, a semi-algebraic condition.

Now, if conditions (27) and (28) are satisfied, we can prove that $\xi_{e, p}$ is continuous. For this let $U_{p} \subset D$ denote the union of all $\left(Y_{i}\right)_{p}$ 's, $i=1, \ldots, t$. This is an open dense subset of $D$ where $\xi_{e, p}$ is continuous. Fix a point $w_{0} \in D$. By (27), $\xi_{e, p}\left(w_{0}\right)$ is the only limit value of $\xi_{e, p}(w)$ for $w \in U_{p}$. Since $\xi_{e, p}$ is bounded, we have

$$
\xi_{e, p}\left(w_{0}\right)=\lim _{\substack{w \rightarrow w_{0} \\ w \in U}} \xi_{e, p}(w)
$$

which means $\xi_{e, p}$ is continuous.

Thus, we obtained a family of continuous maps $\xi_{e, p}$ from $D$ onto $D$, which are holomorphic outside some real analytic locally closed subvariety of codimension 1. By the theorem on removable singularities, $\xi_{e, p}$ is holomorphic on $D$.

Further, by the theorem of Osgood (see [7], Theorem 5, Chapter 5) $\xi_{e, p}$ is biholomorphic if and only if it is injective. This is the condition on fibres:

$$
\#\left(\xi_{e, p}^{-1}(y)\right)=1, y \in D .
$$

The set $\left\{p \in P_{l} \mid\right.$ (30) is satisfied $\}$ is, by Lemma 6.3, semi-algebraic (we set $A:=P^{\prime}, B:=W^{\prime}, C:=D, E:=P^{\prime} \times D \subset A \times B$ and $G$ is the family of graphs of $\left.\xi_{e, p}\right)$. This finishes the proof of Proposition 6.2.

Q. E. D. 


\subsection{The image $\imath\left(A u t_{a}(D)\right)$ and the set of associated graphs.}

Here we wish to prove Proposition 6.1. Let $P_{e, l}$ be the semi-algebraic subsets from Proposition 6.2. We obtain a diagram:

$$
\begin{array}{rcc} 
& & P \\
& \nearrow & \uparrow_{\imath} \\
P_{e, l} & \rightarrow & \operatorname{Aut}_{a}(D) \\
p & \mapsto & \xi_{e, p}
\end{array},
$$

where the map from $P_{e, l}$ into $P$ is the usual inclusion. We define $P_{e, l}^{\prime} \subset P_{e, l}$ to be the subset of all points $p \in P_{e, l}$, for which the diagram is commutative. This condition means $p=\left(v, v^{\prime}, l\right)=\left(v, \xi_{e, p}(v),\left(\xi_{e, p}\right)_{* v}\right)$ and is therefore semialgebraic. Therefore, $P_{e, l}^{\prime}$ is semi-algebraic. The semi-algebraic property of $\imath\left(\right.$ Aut $\left._{a}(D)\right)$ is a consequence of the following observation.

\section{Lemma 6.4}

$$
\imath\left(A u t_{a}(D)\right)=\bigcup_{\substack{e \in E \\ l=1, \ldots, r}} P_{e, l}^{\prime} .
$$

Proof. Let $p \in \imath\left(A u t_{a}(D)\right)$, i.e. $p=\imath(f)$ for some $f \in A u t_{a}(D)$. Then, by Proposition 5.1, $\Gamma_{f} \subset \overline{F_{p}}$. We have $p \in P_{l}$ for some $l=1, \ldots, r$. The graph $\Gamma_{f}$ defines sections in $F$ over every connected open stratum $\left(Y_{i}\right)_{p}, i=1, \ldots, t$. This means that for some choice $e \in E$ we have $f=\xi_{e, p}$. Then $\xi_{e, p} \in \operatorname{Aut}_{a}(D)$, which implies $p \in P_{e, l}$. Further, the equality $f=\xi_{e, p}$ means that diagram (31) is commutative for $p$. Then $p \in P_{e, l}^{\prime}$ and the inclusion in one direction is proven.

Conversely, let $e \in E$ be fixed and $p \in P_{e, l}^{\prime}$. Since $p \in P_{e, l}, f:=\xi_{e, p}$ is an automorphism in $\operatorname{Aut}_{a}(D)$. The commutativity of diagram (31) means $p=\imath(f)$. This implies $p \in \imath\left(\operatorname{Aut}_{a}(D)\right)$, which proves the inclusion in other direction.

Q. E. D.

Proof of Proposition 6.1. The family

$$
\Gamma:=\left\{(\imath(f), w, f(w)) \mid f \in \text { Aut }_{a}(D) \wedge w \in D\right\}
$$

over $P_{e, l}^{\prime}$ coincides now with the family of graphs of $\xi_{e, p}$. The latter is, by construction, semi-algebraic and Proposition 6.1 is proven.

Q. E. D.

\section{Semi-algebraic structures on $A_{u} t_{a}(D)$}

Here we finish the proof of Theorem 1.3. In previous section we considered imbeddings $\imath: \operatorname{Aut}(D) \rightarrow P^{N}$. Here we wish to change to Cartan imbeddings 
$C_{v}(f):=\left(f(v), f_{* v}\right)$. By Proposition 5.1, $\imath$ is given by $\imath(f)=\left(v, f(v), f_{* v}\right)$, where $v=\left(v_{1}, \ldots, v_{m}\right) \in D^{m}$.

The image $C_{v_{j}}\left(A u t_{a}(D)\right)$ is equal to the projection of $\imath\left(A u t_{a}(D)\right)$ on $V_{j}^{\prime} \times L_{j}$ (we use our notations $v_{j}^{\prime}=f\left(v_{j}\right) \in V_{j}^{\prime}, l_{j}=f_{* v_{j}} \in L_{j}$ ). By Theorem 2.1 of Tarski-Seidenberg, $C_{v_{j}}\left(A u t_{a}(D)\right)$ is semi-algebraic. Further, it is semialgebraically isomorphic to $\imath\left(A u t_{a}(D)\right)$. By Remark 5.1, any $v$ and $v^{\prime}$ can be among $v_{j}$ 's. Thus we obtain the following result.

Proposition 7.1 Let $v \in D$ be any point. The image $C_{v}\left(A u t_{a}(D)\right)$ is semialgebraic and this semi-algebraic structure is independent of $v \in D$.

Now we fix some $v \in D$ and denote by $K$ the image $C_{v}\left(A u t_{a}(D)\right) \subset D \times$ $G l(n)$. The family $\Gamma^{\prime} \subset C_{v}\left(A u t_{a}(D)\right) \times D^{2}$ of graphs over $C_{v}\left(A u t_{a}(D)\right)$ is a projection of $\Gamma$ and is therefore semi-algebraic. To simplify our notation we set $P:=D \times G l(n)$, and $\Gamma:=\Gamma^{\prime}$.

Statements 2 and 3 in Theorem 1.3 can now be formulated as follows:

Lemma 7.1 With respect to the group operation of $A u t_{a}(D), K$ is a Nash group and the action on $D$ is Nash.

Proof. We consider the graph of the operation $(x, y) \mapsto x y^{-1}$ in $K^{3}$. For this, start with the family $\Gamma \subset K \times D \times D$ and define a new family $\Gamma_{1} \subset K^{3} \times D^{3}$ by

$$
\Gamma_{1}:=\left\{\left(x, y, z, w, w^{\prime}, w^{\prime \prime}\right) \in K^{3} \times D^{3} \mid\left(y, w^{\prime}, w\right) \in \Gamma \wedge\left(x, w^{\prime}, w^{\prime \prime}\right) \in \Gamma\right\} .
$$

The conditions in (32) express the fact that $y^{-1} \in K$ transforms $w$ in $w^{\prime}$ and $x \in K$ transforms $w^{\prime}$ in $w^{\prime \prime}$. The projection $\Gamma_{2}$ of $\Gamma_{1}$ on $K^{3} \times D^{2}$ (with coordinates $\left(x, y, z, w, w^{\prime \prime}\right)$ ) is, by the Theorem of Tarski-Seidenberg, semi-algebraic. Now the condition $z=x y^{-1}$ means that the graphs of $z$ and $x y^{-1}$ coincide, i.e. the fibres $\left(\Gamma_{2}\right)_{(x, y, z)}$ and $\left(\Gamma_{3}\right)_{(x, y, z)}$ coincide, where $\Gamma_{3}:=\left\{\left(x, y, z, w, w^{\prime \prime}\right) \mid\left(z, w, w^{\prime \prime}\right) \in \Gamma\right\}$ is an extension of $\Gamma$.

By Lemma 6.2, the coincidence of fibres is a semi-algebraic condition on $(x, y, z) \in K^{3}$. This proves that the graph of the correspondence $(x, y) \mapsto x y^{-1}$ is semi-algebraic, which means that the group operation is semi-algebraic. Since the latter is also real analytic by Theorem of Cartan, $K$ is an affine Nash group. Furthermore, the graph $\Gamma$ of the action of $K$ on $D$ is semi-algebraic and real analytic and therefore Nash.

Q. E. D.

It remains to prove statement 1 in Theorem 1.3 which asserts that $A u t_{a}(D)$ is a Lie subgroup of $\operatorname{Aut}(D)$. 
Proof of statement 1. We begin with the semi-algebraic set $K$. By Proposition 2.1, it admits a finite semi-algebraic stratification. Let $x \in K$ be a point in a stratum of maximal dimension. Then there is a neighborhood $U_{x} \subset P$ of $x$, such that $K \cap U_{x}$ is a closed real analytic submanifold of $U_{x}$. The preimage $K^{\prime}:=C_{v}^{-1}\left(K \cap U_{x}\right)$ is a closed real analytic submanifold in the neighborhood $U_{f}:=C_{v}^{-1}\left(U_{x}\right)$ of $f:=C_{v}^{-1}(x)$. Since $\operatorname{Aut}_{a}(D)$ is a subgroup of $\operatorname{Aut}(D)$, we see that $\operatorname{Aut}_{a}(D) \cap\left(f^{-1} \cdot U_{f}\right)=f^{-1} \cdot K^{\prime}$ is a closed real analytic submanifold in

the neighborhood $f^{-1} \cdot U_{f}$ of the unit $i d \in A u t(D)$. This implies that $A u t_{a}(D)$ is a real analytic subgroup of $\operatorname{Aut}(D)$. $\quad$ Q. E. D.

\section{References}

[1] R. Benedetti and J.-J. Risler. Real algebraic and semi-algebraic sets. Actualites Mathematiques. Hermann Editeurs des Sciences et des Arts, 1990.

[2] K. Diederich and J. E. Fornæss. Applications holomorphes propre entre domaines à bord analytique réel. C. R. Acad. Sci. Paris, 307(I):321-324, 1988.

[3] K. Diederich and S. Webster. A reflection principle for degenerate real hypersurfaces. Duke math. journal, 47(4):835-843, 1980.

[4] S. Kaneyuki. On the automorphism groups of homogeneous bounded domains. J. Fac. Sci. Univ. Tokyo, (14):89-130, 1967.

[5] J. J. Madden and C. M. Stanton. One-dimensional Nash groups. Pacific Journal of Math., 154(2):331-344, 1992.

[6] D. Mumford. The red book of varieties and schemes, volume 1358 of Lect. Notes in Math. Springer, 1980.

[7] R. Narasimhan. Several complex variables. Chicago Lectures in Mathematics. Univ. of Chicago Press, 1971.

[8] S. I. Pinčuk. On the analytic continuation of holomorphic mappings. Math. USSR Sbornik, 27(3):345-392, 1975.

[9] R. Remmert and K. Stein. Eigentliche holomorphe Abbildungen. Math. Zeitschr., 73:159-189, 1960.

[10] O. Rothaus. The construction of homogeneous convex cones. Bull. Amer. Math. Soc., (69):248-250, 1963.

[11] E. B. Vinberg, S. G. Gindikin, and I. I. Pyatetskii-Shapiro. Classification and canonical realization of complex bounded homogeneous domains. Trans. Moscow. Math. Soc., (12):404-437, 1963. 
[12] S. Webster. On the mapping problem for algebraic real hypersurfaces. Inventiones math., 43:53-68, 1977.

Dmitri Zaitsev

Fakultät für Mathematik

Ruhr-Universität Bochum

44780 Bochum

Germany

e-mail: Dmitri.Zaitsev@ruba.rz.ruhr-uni-bochum.de 